\title{
Psychological considerations in submarine escape training: brief overview and future directions
}

\author{
Charles H. Van Wijk
}

Institute for Maritime Medicine, Simon's Town, Faculty of Medicine and Health Sciences, Stellenbosch University, South Africa

\begin{abstract}
The inability of a submarine to surface must rate as one of greatest risks to sailors in peacetime. To prepare for such emergencies, many navies provide training to master the procedures required to escape successfully from disabled submarines. This paper provides a brief overview of some of the psychological principles in simulated submarine escape training. It further discusses applicable psychological constructs such as positive outcome expectancies, the role of anxiety, and other personal factors mediating outcomes of such training. It concludes with recommendations for future research aimed at enhancing the safety and impact of submarine escape training. These include enhanced detection of psychological risk factors such as anxiety, as well as investigating the relative contribution of personality variables to in-training safety and positive outcome expectancies. These recommendations do not only apply to submarine escape training, but may also be applicable to high fidelity safety training in other off-shore survival contexts, such as helicopter underwater escape training, freefall lifeboat training and smoke diving.
\end{abstract}

(Int Marit Health 2017; 68, 3: 168-173)

Key words: psychology, anxiety, positive outcome expectancies, pressurised underwater escape, simulator training

\section{HISTORY}

\section{SUBMARINE ESCAPE}

The theme of submarine escape is as old as the use of submarines themselves. The first recorded escape from a submerged submarine was that of Wilhelm Bauer, a corporal in the German Army, and two seamen from the Sea Diver that sank in $18 \mathrm{~m}$ of water in Kiel Harbour on 1 February 1851. Bauer realised that the only way to get out was to raise the internal pressure by flooding the hull with seawater in order to open the hatch. Fortunately the weakly constructed hatches burst open and the men could make good their escape to the surface [1-3]. This incident became the classic forerunner of the accepted routine for escaping from submarines: first a rushed flooding of the pressure hull (or compartment), then a rapid ejection through the hatch, with a free ascent to the surface.

Since Bauer's escape, the technologies in submarines and their escape equipment have developed rapidly. In this, two distinct approaches of surviving a disables submerged submarine developed.

\section{APPROACHES TO SUBMARINE ESCAPE AND RESCUE}

In practice, to exit a submarine immobilised at depth, the inside pressure has to be at or close to ambient pressure, and there are two ways to enact an exit. One way is by direct transfer at the same ambient pressure into a rescue bell or rescue submarine. The other way is emerge from the submarine into the surrounding sea, being exposed to the full pressure of that depth and then float up to the surface. The first way is called submarine rescue, and the second is called submarine escape [4].

The "rescue" model is reliant on an outside agency coming to the rescue of a disabled submarine, and use a Deep Submergence Rescue Vehicle (DSRV) to search for the sunken submarine. If successful, it then locks on to the boat and transfers the submarine's crew to the surface $[5,6]$. However, it is only viable if a DSRV is available.

The "escape" model provides the capability for sailors to leave a disabled submarine without the help of an outside agency $[5,6]$. There are two methods of escape, depend- 
ing on the facilities in a specific boat, and the condition of the boat after becoming unable to surface. Tower escape allows crew members to leave the submarine one at a time, by means of a special airlock, and ascend to the surface under the buoyancy of their submersion suit [7]. This allows escape from depths down to $180 \mathrm{~m}$ [5-8].

Rush escape (also called compartment escape) takes place when no airlock or tower is built into the submarine, or when a submarine's pressure hull is breached and rapid pressurisation occurs inside the boat. The escape compartment (or even the whole pressure hull) is deliberately flooded, while a special trunking under the escape hatch assists the crew to exit the boat and ascent to the surface protected by their immersion suits [7].

Rush escape differs significantly from the tower escape method: in flooding the entire pressure hull, every member of the crew is under pressure all the time during equalisation, increasing the risk for decompression sickness (DCS) following a free ascent.

\section{HEALTH RISKS INVOLVED IN SUBMARINE ESCAPE}

Submarine rescue may be associated with some decompression risk if internal pressure has built up in a disabled boat, but because survivors make their transfer to the surface close to atmospheric pressure, there are relatively few physiological risks involved [4].

In contrast, there are many physiological risks associated with submarine escape, and most revolve around the hyperbaric pressure concerns of decompression and barotraumas. Exposure to a pressurised environment (e.g. during flooding) results in increased absorption of nitrogen in body tissue. When depressurising (e.g. during ascent) the body cannot get rid itself of the nitrogen fast enough, and the nitrogen then form bubbles in the tissue and bloodstream, leading to DCS. Other barotraumas may occur during the rapid compression (e.g. otic barotrauma) prior to escape, or during the rapid decompression (and expansion of air volumes) in the course of a rapid ascent (e.g. pulmonary barotrauma, cerebral arterial gas embolism [CAGE]).

There are numerous other medical dangers associated with submarine accidents and subsequent escape, comprehensively summarised elsewhere $[9,10]$. These centre around the toxic effects of various gasses (from breathing air to battery emissions), panic, barotrauma due to rapid ascents, and DCS - which is dependent on exposure time to the increased pressure inside the hull.

As noted, the extremely high pressure that may be required to equalise the pressure hull to the surrounding seawater increases the risk of gas toxicities as well as DCS. It is therefore essential that the crew members should be under pressure for the shortest possible time before escaping [3, 4].
Effective training ensures that every crew member knows what to do and enable them to do it quickly.

\section{SUBMARINE ESCAPE TRAINING (SET)}

\section{HISTORY}

The first escape simulators ("tanks") to train submariners in the processes and equipment of submarine survival were commissioned in the 1930s [11]. In spite of a decrease in fatal submarine accidents in recent years, training in real-time submarine escape and rescue procedures and equipment remains a priority for all navies with submarine fleets, with international cooperative training exercises held regularly [12].

\section{USE OF SIMULATORS VS. ACTUAL SUBMARINES}

There has been concern that simulator training does not reflect realistic open-sea conditions [13], negating any positive effect from such training. There are indeed a number of documented differences in training escape from a simulator and escape from an actual submarine at sea. For example, escape from a submarine creates a greater physiological stress response and greater adverse changes in mood, and is associated with greater impairment in declarative memory when compared to escape from a simulator [14]. In spite of this, many authors argue that simulated escape training has benefit $[13,15]$, as demonstrated in numerous studies [16-19].

\section{HEALTH RISKS INVOLVED IN ESCAPE TRAINING}

Many of the health risks associated with escape from a disabled submarine are also present during escape training. The main severe medical risks to buoyant ascent escape training in deep simulators are CAGE and/or other pulmonary barotraumas associated with breath holding. Modern developments like facial hoods and survival suits reduced the risk by allowing breathing. It was suggested that wearing survival suits during buoyant ascent escape training reduces the risk for any decompression illness to about 1 in 2500 ascents (or 1 in 1900 for first time trainees) [16]. A recent review of more than 41,000 controlled ascents over 21 years in the Turkish Navy's simulator found no record of pulmonary barotrauma, and middle-ear barotrauma in only $4.1 \%$ of cases (of which half manifested as tympanic membrane rapture), concluding that modern SET is safe [13]. Similarly, a recent review of Unites States Navy data $( \pm 3000$ escapes over 3 years) reported middle ear barotrauma as the most frequent medical incident after pressurised escape training [20].

\section{TRAINING PROTOCOLS}

Generally, SET consists of theoretical and practical components $[19,20]$. Theoretical training includes lectures, and 
offer students the opportunity to familiarise themselves with procedures and equipment. The practical phase typically includes a number of tasks of increased difficulty, culminating in a simulated escape using a purpose build pressurised diving tank or simulator, complete with the applicable pieces of equipment in it. The aim is to facilitate an individual's confidence in their own ability to successfully complete the escape procedures.

From a critical incident training perspective, comprehensive training in the techniques and equipment of submarine escape is essential to minimise the dangers mentioned earlier. A number of principles often guide the training philosophy:

1. Skills based: Trainees learn and practice practical skills (rather than just theoretical knowledge).

2. Graded exposure: Trainees are initially exposed to easier tasks, before being exposed to more difficult tasks in succeeding steps.

3. Stepwise mastery: Trainees continue to each next step only after mastering the tasks of the previous step. These tasks include physiological safety (e.g. equalising ears under pressure, exhaling on ascent), use of equipment (e.g. emersion suit), and coping with the environment (e.g. air pressure, noise, low visibility, etc.) $[19,20]$. One of the more difficult skills to master is to act against natural instincts, namely to exhale on ascent instead of holding their breath [21].

4. Realistic simulation: Research has shown the importance of performing tasks under conditions typical of a real situation, in order to facilitate stimulus generalisation and the transfer of learning [22]. Escape training simulates some of the actual processes on a submarine, and tries to re-create (to the extent possible) some of the conditions of the submarine environment (noise, air pressure, confined space). Further, trainees wear survival suits like they would in the case of an actual escape.

5. Feedback: Positive feedback during simulation training is important in enhancing self-confidence [22]. During SET, students are allowed to progress to every next step only on successful completing of the previous step. This progression then signals success and acts as 'silent' positive feedback on performance.

6. Confidence building: The aim of the training is to instil and enhance confidence. This includes confidence in trainees' knowledge of their equipment, and in their own skills to make good a real escape.

\section{PSYCHOLOGICAL CONSIDERATIONS IN SET}

There are few studies available examining psychological issues in submarine escapes and simulated escape training. However, a number of analogue situations have been studied and reported in the literature, and which provide useful direction when considering SET. Examples include helicopter underwater escape training (HUET) and other offshore survival training processes (e.g. freefall lifeboat training, smoke diving, etc.). Studies on HUET are useful, as it employs the same principles of training, namely a short theoretical phase explaining the principles and processes involved in underwater escape, followed by practical exposure in a simulator, which is usually repeated a number of times. This section will briefly review perspectives from both SET and analogue emergency training experiences.

\section{PSYCHOLOGICAL ISSUES ON A DISTRESSED SUBMARINE}

Two studies examined psychological issues in simulated submarine accidents, and highlighted a number of issues that might affect crew members' ability to escape (including decision making and levels of anxiety). In a survival trial of 7 days in a simulated distressed submarine, the associated cold led to poor sleep and progressive difficulty in sustained concentration (in comprehension and memory) [23]. In a week-long disabled submarine exercise, emotional stress was highest in the first $24 \mathrm{~h}$, improving over the rest of the time in confinement. Levels of distress were further related to hardiness and other personality traits, suggesting that personality variables may also influence the experience of submariners during escape training [24].

\section{PSYCHOLOGICAL ISSUES AFTER SUBMARINE ACCIDENTS}

In the aftermath of actual disasters at sea, exposed crew members reported more post-traumatic stress symptoms 2 weeks after non-fatal submarine accidents, compared to non-exposed controls, but less than surface ship survivors [25]. Problem-focussed coping facilitated good adjustment, while previous exposures to traumatic events were associated with greater symptom reporting. Less acute stress response symptoms were reported where submariners were satisfied with their coping behaviour. This suggests that if good training can lead to an expectation of positive coping, this may generalise to real life and facilitate good adjustment after accidents [25]. Elsewhere, 9\% of survivors met criteria for post-traumatic stress disorder (PTSD) 7 months after a traumatic submarine accident. There were anecdotal reports that the low levels of PTSD was because of the good training for disasters among the submarine's crew, which highlights the importance of platform-specific disaster training (i.e. submarines per se) [26].

\section{PSYCHOLOGICAL ASPECTS OF SAFETY TRAINING}

Positive outcome expectancies (POE). Studies on psychological aspects of safety training are unanimous that the development of positive response-outcome expectancies is 
the main (and critical) training effect of such programmes [16-19]. This fits well with submariners' reports that confidence in their ability to control risk is a strong protective factor in coping during underwater missions [27]. The main psychological obstacle in developing positive response-outcome expectancies appear to be panic and other forms of overwhelming anxiety during training.

Anxiety. Training for emergencies (i.e. 'simulated exposure') precipitates a raise in state anxiety [16-19]. This peaks with the onset of practical evolutions [21], with a significant decrease in anxiety after completion of the practical evolutions [19]. Non-swimmers reports greater pre-course anticipatory anxiety [18]. The increase in anticipatory anxiety can be observed in both physiological and psychological markers. Significant increases in saliva cortisol has been reported $[17,18]$, and physiological manifestations of anxiety could also manifest in visible symptoms such as shivering and hyperventilation. Psychologically, increased anxiety was reported using scales such as the STAI and IPAT Anxiety Scale [16-19], compared to baseline data. The Yerkes-Dodson law (1908) posits that performance increases with physiological or mental arousal, but when levels of arousal become too high, performance decreases (the so-called inverted U-curve). Therefore, in contrast to mild anxiety, excessive anxiety may have a negative effect on simulator performance, thus interfering with the potential benefit of training by lowering POE (i.e. confidence in future ability to handle such situations). In summary, during highrisk safety training, anxiety has an effect on two levels: firstly it affects performance during training, which in turn lowers confidence, thus frustrating the aim of training; secondly it compromises the individual's safety during the training. Highly anxious candidates may require closer monitoring.

Training outcomes and mediating factors. The desired outcome of safety training is a positive, or at least increased, response-outcome expectancy. Training has been shown to increase confidence in specific contexts like the use of helicopter transport [18] and submarine operations [28], but also increase confidence in generalised POE and coping $[16,18]$. General coping through safety training develops through repetition and controlled action [18]. While increased outcome expectancies are psychological in nature, positive outcomes also happen on a physiological level. For example, anxiety induced heart rate response to HUET is reduced after the first practical evolution, and combined heart rate response to HUET is habituated after 4 trials [29]. This points to the value of repetitive training in habituating physiological response, and also to the need of enough trials to achieve this. Training thus needs multiple exposures in close succession to achieve optimal effect.

Multiple personal factors have been found to influence the effect of training on outcome. Not surprisingly, previous safety training was associated with lower anxiety during HUET training, as was previous diving experience [18]. The benefit of previous diving experience for SET may lie in that previous exposure to increased air pressure and/or exposure to enclosed suits (e.g. wetsuit) may reduce anxiety, thus enhancing the effect of training. As noted, non-swimmers reported greater pre-course anticipatory anxiety [16], and water orientation for non-swimmers is recommended, as the discomfort of non-swimmers, once in the water filled simulator, contributes to increased anxiety.

Personality variables have also been found to mediate the effect of training on outcome expectancies. Higher trait anxiety was associated with lower confidence in helicopter flying (after HUET) and lower satisfaction with personal coping [18]. It has been hypothesised that high anxiety prevents the development of positive response outcome expectancy by interfering with the cognitive functions needed during training. Further, training may be of too short duration to achieve the prolonged exposure required for decreasing anxiety, and it has been recommended that higher anxiety candidates may benefit more from systematic desensitisation [30] than exposure therapy as intervention of choice.

Trainees who report more external locus of control also have a greater physiological stress response (i.e. higher saliva cortisol) [18], suggesting greater autonomic activation and associated poorer training performance. However, studies examining associations between locus of control and anxiety and training performance remain inconclusive [16, 21]. In the same way, studies on the influence of risk-taking (measured by the Sensation Seeking Scale) on training outcomes also remain inconclusive [16].

An unpublished study found dispositional confidence (measured by the 16PF Factor 0 ) significantly correlated to lower anxiety and more positive outcomes during SET. It was hypothesised that students with high self-confidence would probably appraise the training as less threatening, which would result in less anticipatory anxiety, which in turn could contribute to higher confidence in their knowledge and equipment. Whatever the mechanism, high scores were considered a protective factor during escape training [28].

Stress inoculation training [31] has been shown to facilitate POE during safety training [17], which may provide a validated intervention approach to enhance training outcomes. A more recent development is the introduction of mindfulness training in the SET context [32]. Trainees with higher mindfulness scores reported less anxiety during training, and also displayed less cognitive degradation, although there was no difference between higher and lower mindfulness scores on their mood state scores during training [32].

Lastly, realistic simulation (so-called "high fidelity" training) is only effective when trainees perceive the experience as successful [22]. In this regard the role of positive 
feedback (of success) is critical [22]. Stepwise progression - where a candidate only continues to the next step after mastery of the previous one - is one way of achieving this. Being allowed to progress becomes the positive feedback of success at each step.

\section{DIRECTIONS FOR FUTURE RESEARCH}

Questions regarding the relative contribution of psychological and/or other biographical variables to SET outcomes remain. These may have important practical implications, as it relates to how psychological variables mediate the effect of SET on POE. Further research is needed to answer these questions and inform best practices for SET.

State anxiety. Excessive state or anticipatory anxiety has been shown to pose risks to a successful outcome of SET. Accurate detection of significantly elevated state anxiety could alert simulator instructors to the risk of it interfering with either safety during training, or the development of POE. Studies are needed to determine the best ways to measure this, which may include self-report measures, physiological markers, and observers' reports. Analyses could include correlating such measures with performance during pressurised escapes and with longer term outcome expectancies. Such studies could inform the possible introduction of psychological measures, additional to the physical examinations which are required of trainees prior to commencing with escape training. Further, it could inform simulator instructors of trainees that require additional preparation, before embarking on the practical pressurised escape.

Intervention/preparation. If individuals with excessive anxiety can be identified, well controlled studies would be required to determine the best way to respond to it, as well as considering whether there would be value in providing additional anxiety management training to all individuals prior to high fidelity safety training. In particular, studies are needed to test which short-term interventions are best suited to reduce anxiety and facilitate positive training outcomes. For example comparison studies of group interventions (e.g. half-day stress inoculation training vs. other forms of cognitive behaviour therapy training vs. mindfulness training, etc.).

Water orientation. The effect of perceived water orientation and/or swimming ability on anxiety, in-training performance, and development of POE require further clarification. Firstly, it may be useful to determine its relative contribution to outcomes, and if found significant, studies will be needed to identify what form of preparatory water orientation would be appropriate, and how to offer it.

Personality variables. With personality variables shown to influence the impact of safety training, investigating the relative contribution of such variables may add value to the development of POE. Studies which include measures for multiple constructs, such as trait anxiety, locus of control, risk-taking, and dispositional confidence, among others, are needed to tease out the relative contribution of these variables to training outcomes. This could include, for example, studies correlating measures of personality traits with medical incidents during training. Other studies could correlate personality trait measures with measures of POE after completion of the training.

1. Trait anxiety has been used extensively in studies involving underwater activities. Self-report trait anxiety measures could predict in over $80 \%$ of panic events among scuba students [33] and experienced scuba divers [34], and holds promise to identifying at-risk candidates and thus facilitate enhanced preparation.

2. Locus of control. Given the inconsistent evidence, and the hypothesised associations between internality-externality scores and the development of POE [35], studies are needed to clarify possible mediating effects of locus of control on SET outcomes.

3. Risk-taking. Risk-taking or sensation seeking as personality traits have been reported as relevant to other safety-critical training (e.g. military diving [36]). Its role in facilitating simulator performance during SET remains unclear and studies are needed to clarify possible mediating effects on SET outcomes.

4. Dispositional confidence. Safety training aims to enhance individuals' confidence in their ability to successfully manage real emergencies. Trainees with high dispositional confidence may therefore emerge from training with different gains than those with lower dispositional confidence. Studies are needed to clarify the relative contribution of general self-confidence on training outcomes, to optimise the training experience for those who require more input. Closely associated with self-confidence is the role of confidence in key figures involved in the management of emergencies on board submarines. Although no research could be located examining the role of such figures in maintaining confidence and POE in real emergencies, including their contribution in studies investigating high-fidelity training could be useful in translating findings to real life conditions on board operational submarines.

\section{CONCLUSIONS}

Simulated training in submarine escape procedures are done according to well develop principles, whose value in achieving POE have been demonstrated. Given the demanding nature and continued implementation of such training, there is still room for improvement. The previous section put forward a number of areas that could assist in focussing tailored studies that could provide answers that 
may enhance the positive outcomes of SET. Navies offering simulated SET are therefore encouraged to embark on such studies with the aims to contribute to increased safety and efficacy of such training. This may have the added benefit that enhanced insights from SET studies could potentially also be generalised to other high fidelity safety training experiences in the field of off-shore survival, such as helicopter underwater escape training, freefall lifeboat training and smoke diving.

\section{REFERENCES}

1. Adkisson G. Submarine Escape and Rescue. SPUMS J. 1990; 20(4): 220-222.

2. Shelford WO. "Subsunk". London: George G Harrap \& Co Ltd London, 1960.

3. Tabb HJ. Escape from Submarines: A Short Historical Overview of Policy and Equipment in the Royal Navy. R I N A Transactions. 1975; 117: 19-39.

4. Elliott D. A short history of submarine escape: the development of an extreme air dive. SPUMS J. 1999; 29(2): 81-87.

5. O'Brien G. Submarine escape: Where now? Nav Int. 1987; 92(9): 482-484.

6. O'Brien G. The Great Escape. Defence. (1988) https://archive.is/ /BK8JG.

7. White MG. Submarine escape and rescue. Naval Forces. 1990; 11(1): 41-47.

8. Turner MS. Submarine escape from a depth of 300 feet: a personal experience. J R Nav Med Serv. 1995; 81(3): 210-213, indexed in Pubmed: 8736316.

9. Walker RM. A. complete submarine escape and rescue organisation. SPUMS J. 1997; 27(2): 95-101.

10. Walker R. Submarine Medicine. In: Edmonds C, Lowry C, Pennefather J, Walker R, editors. Diving and Subaquatic Medicine, 4th ed. New York: Hodder-Arnold. Diving Subaquatic Med. 2002: 665-670.

11. Moses H. Casualties in individual submarine escape. Groton, Conn.: US Naval Submarine Medical Centre Groton, 1964.

12. International Submarine Escape and Rescue Liaison Office. http:// www.ismerlo.org/ (2017).

13. Yildiz S, Ay H, Günay A, et al. Submarine escape from depths of 30 and 60 feet: 41,183 training ascents without serious injury. Aviat Space Environ Med. 2004; 75(3): 269-271, indexed in Pubmed: 15018296.

14. Trousselard M, Cian C, Barraud PA, et al. Physiological and psychological effects of escape from a sunken submarine on shore and at sea. Aviat Space Environ Med. 2009; 80(10): 850-856, doi: 10.3357/asem.2503.2009.

15. Knight J, Williams GA. medical review of emergency ascent training. SPUMS J. 1993; 23(4): 230-236.

16. Harris RA, Coleshaw SRK, MacKenzie IG. Analysing stress in offshore survival course trainees. Aberdeen: Health and Safety Executive, 1996. Offshore Technology Report: OTH 94 446. http://www.hse. gov.uk/research/othpdf/400-499/oth446.pdf (1996).

17. Hytten K, Jensen A, Skauli G. Stress inoculation training for smoke divers and free fall lifeboat passengers. Aviat Space Environ Med. 1990; 61(11): 983-988, indexed in Pubmed: 2256886.
18. Hytten K, Jensen A, Vaernes R. Effects of underwater escape training-a psychophysiological study. Aviat Space Environ Med. 1989; 60(5): 460-464, indexed in Pubmed: 2730491.

19. van Wijk C. Submarine escape: the effect of training on anxiety. Mil Med. 1998; 163(2): 68-70, indexed in Pubmed: 9503894.

20. O'Donnell SW, Horn WG. Initial review of the U.S. Navy's pressurized submarine escape training outcomes. Undersea Hyperb Med. 2014; 41(1): 33-40, indexed in Pubmed: 24649715.

21. Blake D. The Great Escape. Navy News 1997: 12-13.

22. Keinan G. Training for dangerous task performance: The effect of expectations and feedback. J AppI Soc Psychol. 1988; 18(4): 355-373.

23. Slaven GM, Windle CM. Cognitive performance over 7 days in a distressed submarine. Aviat Space Environ Med. 1999; 70(6): 604-608, indexed in Pubmed: 10373054.

24. Eid J, Johnsen BH, Saus ER, et al. Stress and coping in a week-long disabled submarine exercise. Aviat Space Environ Med. 2004; 75(7): 616-621, indexed in Pubmed: 15267084.

25. Eid J, Johnsen BH. Acute stress reactions after submarine accidents. Mil Med. 2002; 167(5): 427-431, indexed in Pubmed: 12053854.

26. Berg JS, Grieger TA, Spira JL. Psychiatric symptoms and cognitive appraisal following the near sinking of a research submarine. Mil Med. 2005; 170(1): 44-47, doi: 10.7205/milmed.170.1.44, indexed in Pubmed: 15724853.

27. Kimhi S. Understanding Good Coping: A Submarine Crew Coping with Extreme Environmental Conditions. Psychol. 2011; 02(09): 961-967, doi:10.4236/psych.2011.29145.

28. Institute for Maritime Medicine. The Effect of Submarine Escape Training on Anxiety and Confidence. Simon's Town: South African Military Health Service; 1999. Technical Report, August 1999.

29. Tipton MJ, Gibbs P, Brooks C, et al. ECG during helicopter underwater escape training. Aviat Space Environ Med. 2010; 81: 399-404, doi: 10.3357/ASEM.2700.2010.

30. Brooks CJ, Gibbs PN, Jenkins JL, et al. Desensitizing a pilot with a phobic response to required helicopter underwater escape training. Aviat Space Environ Med. 2007; 78(6): 618-623, indexed in Pubmed: 17571665.

31. Saunders T, Driskell JE, Johnston JH, et al. The effect of stress inoculation training on anxiety and performance. J Occup Health Psychol. 1996; 1(2): 170-186, doi: 10.1037//1076-8998.1.2.170, indexed in Pubmed: 9547044.

32. Trousselard M. Escape from a Diving Submarine Simulator: Impacts of Mindfulness Differences on Physio-Biological Responses and Cognitive Performances. Paper presented at the RTO Human Factors and Medicine Panel Symposium Bulgaria, 2009.

33. Morgan WP, Raglin JS, O'Connor PJ. Trait anxiety predicts panic behavior in beginning scuba students. Int J Sports Med. 2004; 25(4): 314-322, doi: 10.1055/s-2004-815829, indexed in Pubmed: 15162252.

34. Colvard DF. Identifying anxiety and panic risk in divers. Paper presented at DAN-SA Diver Stress and Panic Prevention Workshop. Johannesburg, South Africa, 2007 Sep 27.

35. Carver C. The Internal-External Scale Confounds Internal Locus of Control with Expectancies of Positive Outcomes. Pers Soc Psychol Bull. 1997; 23(6): 580-585, doi: 10.1177/0146167297236002.

36. Van Wijk C, Fourie M. Using psychological markers of sport injuries for navy diving training. Int J Sport Exerc Psychol. 2015; 15(1): 1-11, doi: 10.1080/1612197x.2015.1056903. 